\title{
Stellar models for a wide range of initial chemical compositions until helium burning ${ }^{\star}$
}

\section{From $X=0.55$ to $X=0.75$, for $Z=0.03$}

\author{
A. Claret \\ Instituto de Astrofísica de Andalucía, CSIC, Apartado 3004, E-18080 Granada, Spain
}

Received October 28, 1996; accepted January 24, 1997

\begin{abstract}
In this Paper I present grids for the stellar models with a slightly higher metallic content than in the previous works (Claret 1995; Claret \& Giménez 1995), say, $Z=0.03$. The initial helium abundances in mass are $Y_{\mathrm{i}}=0.42,0.32$ and 0.22 ; this last value was used only to facilitate interpolations since it is a little bit smaller than the primordial helium abundance. The present computations are based on the radiative opacities with spin-orbit coupling provided by the Lawrence Livermore group (Iglesias et al. 1992). For the lower temperatures I have used the results by Alexander (1992). Core overshooting was taken into account as well as mass loss. The models presented here cover the mass range between 1 and $40 M_{\odot}$. I also compute for all models the internal structure constants $k_{j}$ and the radius of gyration $\beta$. For the first time the calculation of the tidal constants $E_{2}$ and $\lambda_{2}$, which are used to evaluate circularization and synchronization times in binary stars, are presented for stellar models as a function of the initial mass and time. The former is related with the dynamical tidal contribution to the total perturbed potential in a binary star while the latter is connected with the external structure of the outer layers.
\end{abstract}

Key words: stars: binaries: close; evolution; interiors; fundamental parameters; abundances

\section{Introduction}

As in this Paper I present the results for stellar models which differ from previous papers of this series essentially in the chemical composition the description of the physics input will be not repeated here. The main characteristics

Send offprint requests to: A. Claret, e-mail:claret@iaa.es

* Tables 1-72 are only available in electronic form at the CDS via anonymous ftp to cdsarc.u-strasbg.fr (130.79.128.5) or via http://cdsweb.u-strasbg.fr/Abstract.html and the justification for the stellar models presented are described in Claret 1995 (Paper I). Besides the difference in the initial chemical composition, I also compute the tidal constants $E_{2}$ and the tidal coefficients $\lambda_{2}$ for a few models of these grids. In the next papers of this series these calculations will be available for all models. Meanwhile I can provide specific calculations under request. Such parameters are very important in order to evaluate the critical times for circularization and synchronization in close binary systems (CBS) (see Claret \& Cunha 1996).

\section{The grids and the tidal friction in close binary systems}

\subsection{The models}

The mass range covered is from 1 to $40 M_{\odot}$ for the three chemical compositions $(X, Z)=(0.55,0.03),(0.65,0.03)$ and $(0.75,0.03)$. I denote these grids hereafter as X55, X65 and X75 respectively. Note that for X75, the abundance of helium is 0.22 . This value is a little bit smaller than that used as primordial abundance of helium (about $0.24)$. However, this grid is useful to permit interpolations among stellar properties and isochrones as explained in the Introduction of the paper Claret \& Giménez 1995. The value of $Y$ for the models $\mathrm{X} 65$ is obtained by the relation $Y=Y_{\mathrm{p}}+(\Delta Y / \Delta Z) Z$, withY $\mathrm{p}_{\mathrm{p}}$ (the primordial $\mathrm{He}$ abundance) and $\Delta Y / \Delta Z$ taken equal to 0.24 and 2.7 respectively; two other values of $Y$ were considered to take into account the uncertainties in $Y_{\mathrm{p}}$ and in the derivative $\Delta Y / \Delta Z$. In Figs. 1-6 I show the HR and $\log T_{\text {eff }} \times \log g$ diagrams. Figures 4,5 and 6 are especially useful to be compared with binary star data given that the masses and radii in this kind of stars are generally the best determined parameters.

From the comparison among these figures one can note the influence of the mean molecular weight on the position of the tracks. Let us use simple homology relations, for example ideal gas and constant opacity, to interpretate such 
diagrams. Under such conditions, the radius of a model is proportional to $\mu^{\frac{\nu-4}{\nu+3 \lambda}}$ where $\mu$ is the mean molecular weight, $\lambda$ is the exponent of the density and $\nu$ is the exponent of the temperature in the nuclear energy generation rate. For the pp chain $\nu \approx 4-5$, while $\lambda=1$. For a fixed mass, it is easy to see that the models X55 - large mean molecular weight - have larger radii and smaller $\log g$ than the corresponding X75 grid. The aspect of Fig. 7 can be interpretated using the following argument: let be $f \mathrm{XM}$ the fraction of the stellar mass for which hydrogen is converted into helium during the lifetime of the star, being $f$ smaller than $1, X$ is the initial hydrogen content per mass and $M$ the total mass. The corresponding lifetime in the main-sequence will be given by the ratio of the total energy radiated to the average luminosity. In this way, $\tau \propto$ $f \mathrm{XM} / \mathrm{L}$. A more direct comparison concerning the influence of the molecular weight on the tracks can be seen in Fig. 7 where the lifetimes of the hydrogen burning is shown for the three grids as a function of the logarithm of the stellar masses. An increase of 0.10 in the initial hydrogen content implies that the corresponding lifetimes are increased by about 1.7 times. On the other hand, Fig. 8 shows the effects of changing the chemical composition on the effective temperatures for the models at TAMS, also as a function of the logarithm of the stellar masses. Note that a variation of 0.10 in the initial helium abundance yields a difference about 0.08 in $\log T_{\text {eff }}$.

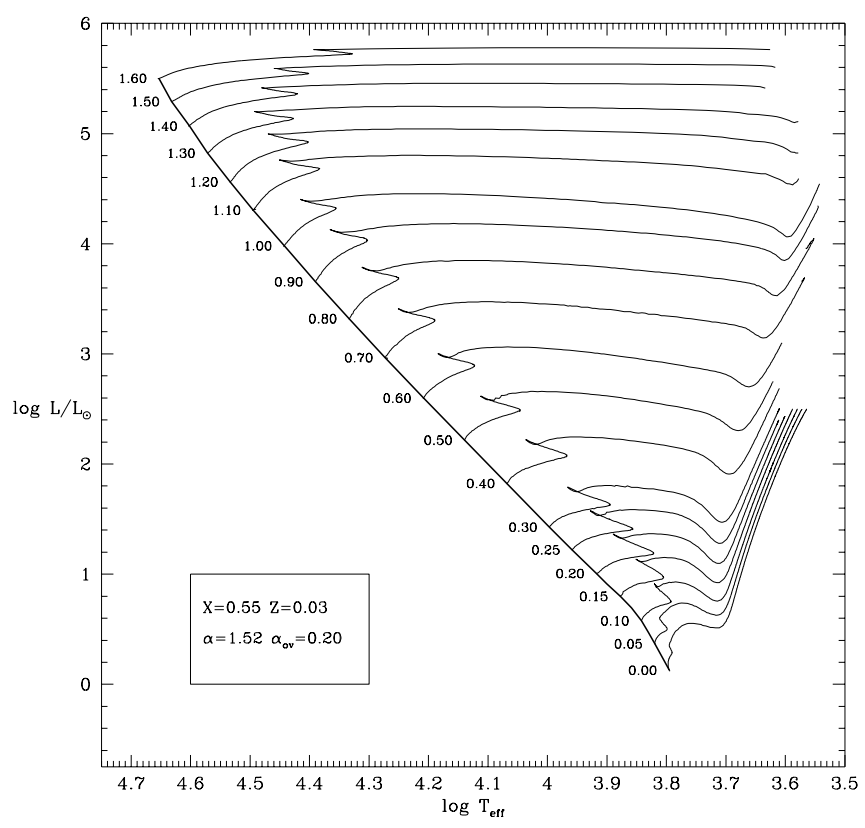

Fig. 1. HR diagram for the grid X55. Numbers attached denote $\log M$

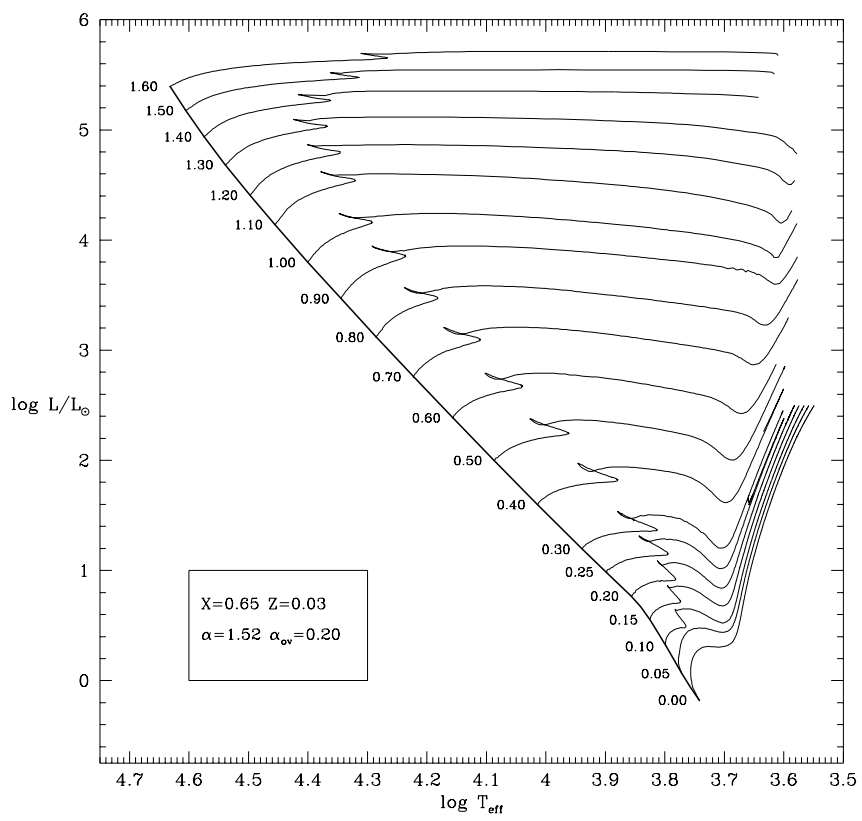

Fig. 2. HR diagram for the grid X65. Same remarks a Fig. 1

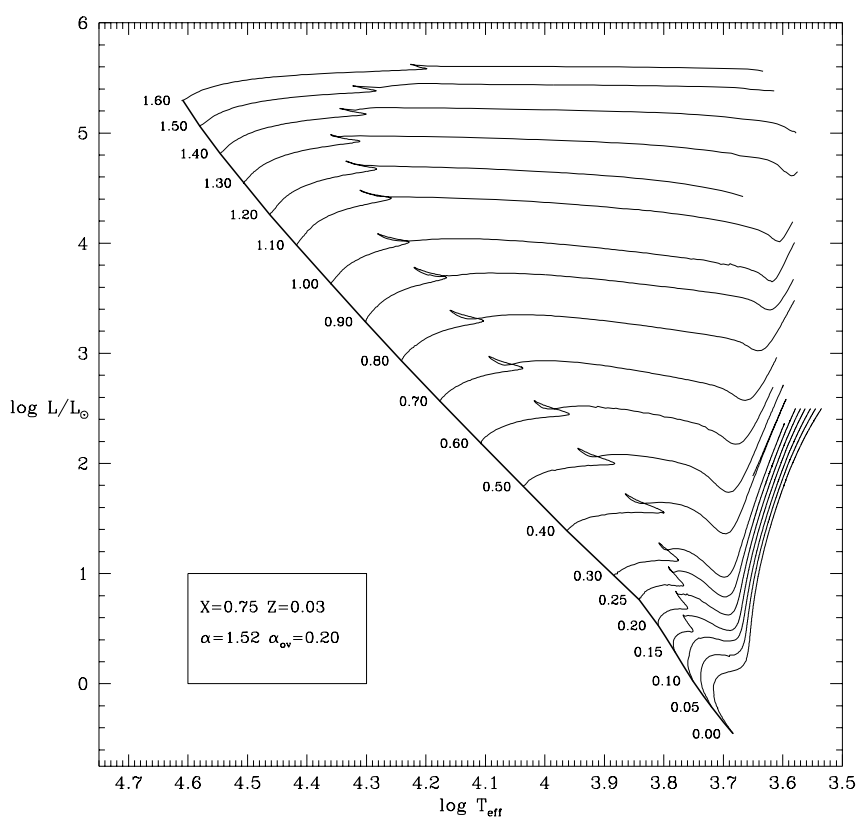

Fig. 3. HR diagram for the grid X75. Same remarks as Fig. 1

\subsection{Tidal evolution}

Concerning tidal evolution, there are three mechanisms which are being used to study the circularization and synchronization of orbits in CBS. The first two mechanisms, which are based on the classical tidal theory, depend on the characteristics of the stellar core and envelope. On the other hand, Tassoul claimed that the distortions present in CBS cause hydrodynamical currents which tend to circularize their orbits. However, there is a strong discussion on 


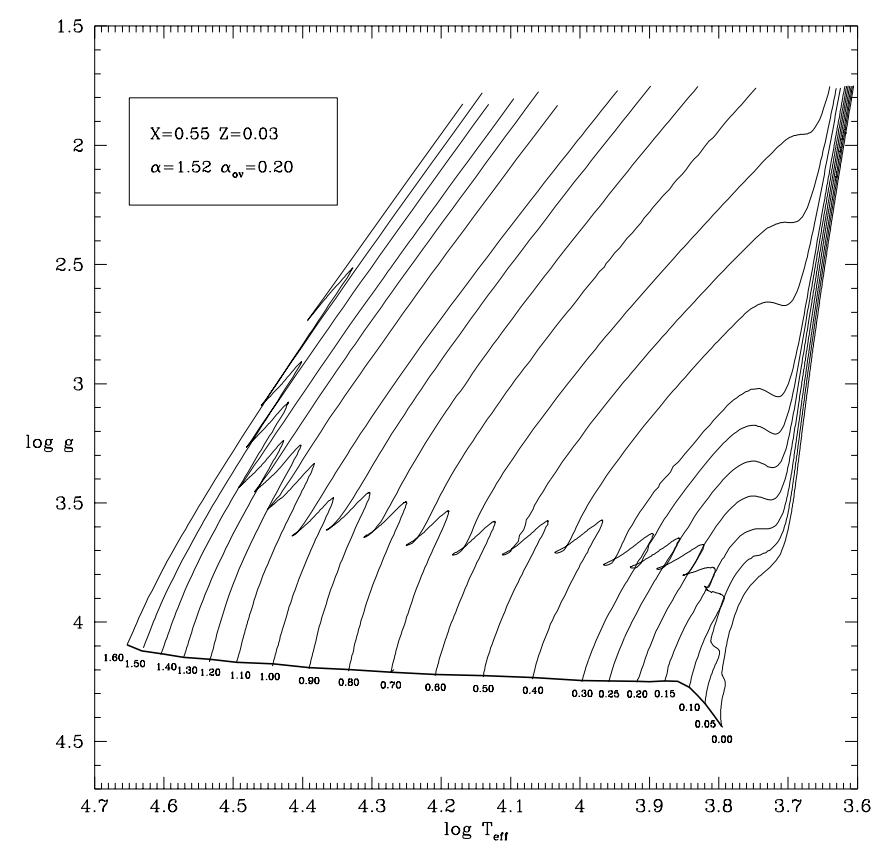

Fig. 4. $\log g-\log T_{\text {eff }}$ diagram for the grid X55. Same remarks as Fig. 1

the validity of the hydrodynamical mechanism (Rieutord 1992; Tassoul \& Tassoul 1996). I do not discuss here this controversy. The interested readers can consult the original papers quoted above. For a comparison of predictions of the hydrodynamical and tidal-torque mechanisms with observations see Claret et al. (1995) and Claret \& Cunha (1996).

I shall concentrate our attention on the first two mechanisms. A star which belongs to a binary system is under the action of the disturbing potential due to its mate. This star reacts to the external potential through equilibrium and dynamical tides. The characteristic time scales for synchronization and circularization in the case of turburlent dissipation and radiative damping are given respectively by (see for example Zahn 1975 and 1989)

$$
\begin{aligned}
& \left(\tau_{\text {sync }}\right)_{\text {turb }}{ }^{-1}=-\frac{1}{\Omega-\omega} \frac{\mathrm{d} \omega}{\mathrm{d} t} \\
& =3.9510^{2} \beta^{2} M^{7 / 3} \frac{(1+q)^{2}}{q^{2}} L^{-1 / 3} \lambda_{2}{ }^{-1} \frac{P^{4}}{R^{16 / 3}} \\
& \left(\tau_{\text {circ }}\right)_{\text {turb }}{ }^{-1}=-\frac{1}{e} \frac{\mathrm{d} e}{\mathrm{~d} t} \\
& =1.9910^{3} M^{3} \frac{(1+q)^{5 / 3}}{q} L^{-1 / 3} \lambda_{2}{ }^{-1} \frac{P^{16 / 3}}{R^{22 / 3}} \\
& \left(\tau_{\text {sync }}\right)_{\mathrm{rad}}-1=-\frac{1}{\Omega-\omega} \frac{\mathrm{d} \omega}{\mathrm{d} t} \\
& =2.03 \beta^{2} M^{7 / 3} \frac{(1+q)^{2}}{q^{2}} E_{2} \frac{P^{17 / 3}}{R^{7}}
\end{aligned}
$$

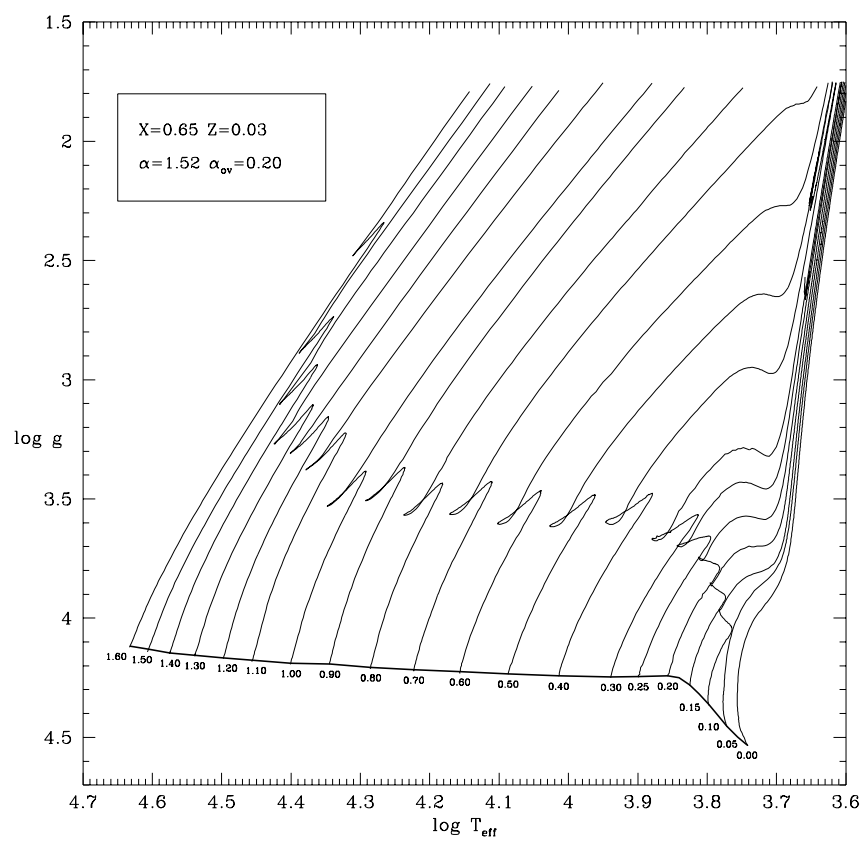

Fig. 5. $\log g-\log T_{\text {eff }}$ diagram for the grid X65. Same remarks as Fig. 1

$$
\begin{aligned}
\left(\tau_{\text {circ }}\right)_{\mathrm{rad}}^{-1} & =-\frac{1}{e} \frac{\mathrm{d} e}{\mathrm{~d} t} \\
& =1.7110^{1} M^{3} \frac{(1+q)^{5 / 3}}{q} E_{2}{ }^{-1} \frac{P^{7}}{R^{9}}
\end{aligned}
$$

where the times scales are in years, the orbital period $P$ in days and the mass, radius and luminosity are in solar units. The subscripts circ and sync refer to circularization and synchronization while turb and rad refer to turburlent dissipation and radiative damping respectively. The rotational angular frequency is represented by $\Omega$ while $e$ is the eccentricity of the orbit, $q$ the mass ratio, $\beta$ the radius of gyration and $\omega$ is the orbital angular frequency. The meaning of $E_{2}$ and $\lambda_{2}$ will be explained in the next paragraphs.

The few papers that treat the oscillations driven by the tides do not consider the dissipation in the mentioned process (e.g. Cowling 1941). In more recent years Zahn (1975) studied the effects of the radiative damping in the dynamical tide. This mechanism, radiative damping, is the responsible for the synchronization and circularization of the orbits when the stars in a given system present convective cores and radiative envelopes. The gravity waves, due to the external potential, are damped in the external layers. This damping occurs because the radiative cooling time is of the order of the tidal period and in this way part of the angular momentum of rotation is transfered to the orbital motion. In order to characterize the time scales I use $E_{\mathrm{n}}$. Such a parameter can be evaluated using 


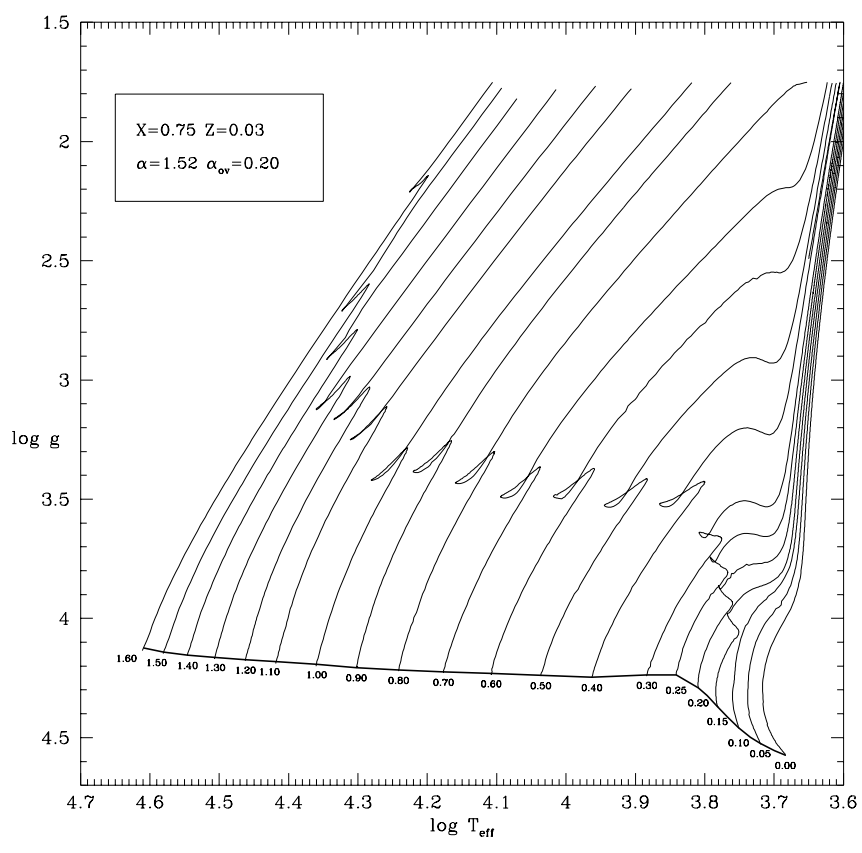

Fig. 6. $\log g-\log T_{\text {eff }}$ diagram for the grid X75. Same remarks as Fig. 1

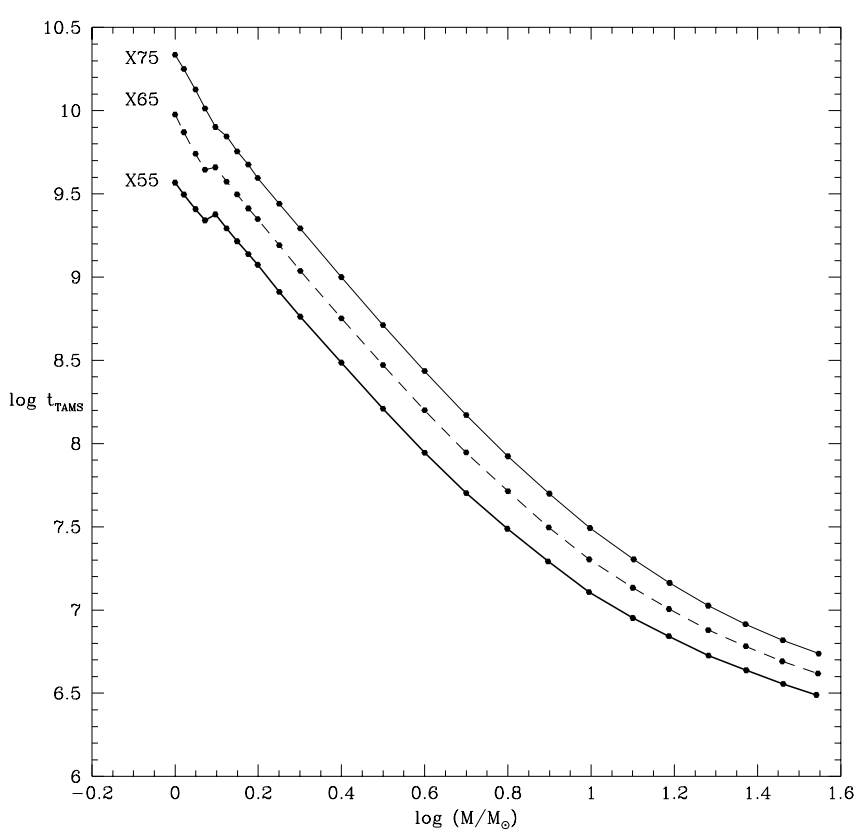

Fig. 7. The lifetimes for the hydrogen-burning phase for the three grids. The grid X55 is represented by a thick continuous line, X65 by a dashed one, and the continuous thin line denotes the grid X75

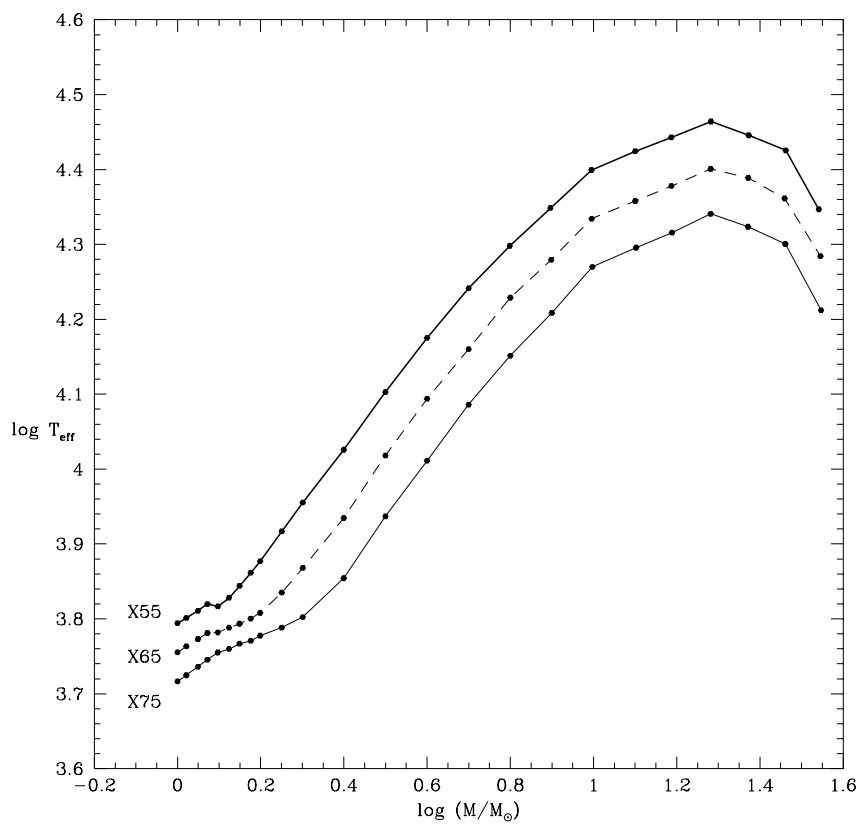

Fig. 8. The effective temperatures at the TAMS for the three grids. Same line types as in Fig. 7

the following equations:

$$
\begin{aligned}
E_{\mathrm{n}}= & \frac{3^{8 / 3}(\Gamma(4 / 3))^{2}}{(2 n+1)[n(n+1)]^{4 / 3}} \frac{\rho_{f} R^{3}}{M} \\
& {\left[\frac{R}{g_{s}}\left(\frac{-g B}{x^{2}}\right)_{f}^{\prime}\right]^{-1 / 3} H_{\mathrm{n}}^{2} }
\end{aligned}
$$

where $\Gamma$ is the gamma function, $x$ is the normalised radius of the configuration, the symbol $f$ denotes the border of the convective core, $s$ indicates surface values, the prime denotes the derivative with respect to $x, R$ is the radius, $M$ the mass, $g$ the gravity and $B$ is given by

$B=\frac{\mathrm{d}}{\mathrm{d} r} \ln \rho-\frac{1}{\Gamma_{1}} \frac{\mathrm{d}}{\mathrm{d} r} \ln P$

where $-g B$ is the square of the Brunt-Väisälä frequency.

The coefficient $H_{\mathrm{n}}$ is given by

$H_{\mathrm{n}}=\frac{1}{X\left(x_{f}\right) Y(1)} \int_{0}^{x_{f}}\left[Y^{\prime \prime}-\frac{n(n+1) Y}{x^{2}}\right] X \mathrm{~d} x$

$X$ is the solution of the differential equation

$$
X^{\prime \prime}-\frac{\rho^{\prime}}{\rho} X^{\prime}-\frac{n(n+1)}{x^{2}} X=0
$$

and $Y(1)$ is the solution of the Clairault equation. The differential equations were solved for each configuration using a fourth order Runge-Kutta method. In this Paper I shall restrict the calculations to $n=2$ since for larger $n$ the contribution to the dynamic tide is very small. Such 
calculations deserve special attention. Let us rewrite $-g B$ as

$-g B=\frac{G^{2} M_{r}^{2}}{r^{4} P}\left[\frac{4-3 \beta_{1}}{\beta_{1}}\left(\nabla_{\mathrm{ad}}-\nabla\right)+\nabla \mu\right]$

where $\beta_{1}$ is the usual $P_{\text {gas }} / P$ ratio, $G$ is the constant of gravitation, $r$ the radial distance, $M_{r}$ the mass at the distance $r, P$ the total pressure, $\nabla \equiv \mathrm{d} \ln T / \mathrm{dnl} P, \nabla$ ad is the adiabatic gradient and $\nabla \mu \equiv \operatorname{dln} \mu / d \ln P$. As a massive star evolves the convective core recedes. The zone between the initial and the present convective core present a large chemical compositon gradient. In such zone the spatial gradient of chemical composition increases more and more and becomes wider. This feacture is well known when one analyses a diagram of propagation. As shown before, the tidal constant depends on the derivative of the Brunt-Väisäla frequency in the border of the convective core and the consequent numerical oscillations are clear in Fig. 9.

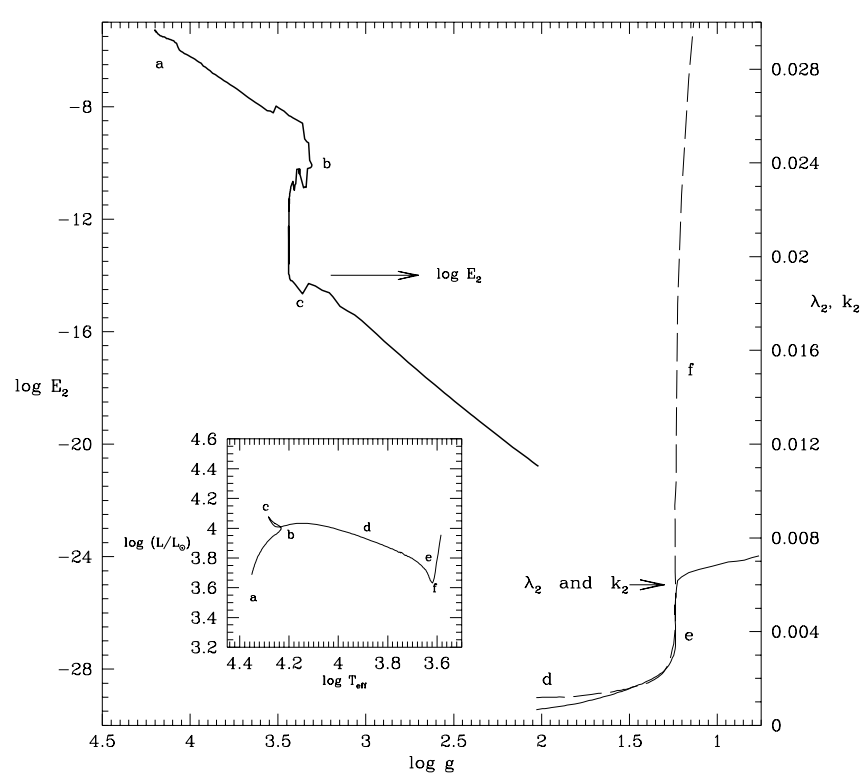

Fig. 9. Schematic evolution of $E_{2}$ (thick line) and $\lambda_{2}$ (thin line) for a $10 M_{\odot}$ model with $X=0.75$ and $Z=0.03$. The apsidal motion constant is denoted by a dashed line. The attached labels are useful to identify the corresponding evolutionary phases since that in the lower left corner I represent the corresponding HR diagram

For stars with envelopes in convective equilibrium another mechanism was identified: the turbulent dissipation. This process is characterized by the parameter $\lambda_{2}$ though in the last years the apsidal motion constant $k_{2}$ was used as an approximation. In fact, Fig. 3 by Claret \& Cunha (1997) shows that it is an acceptable approximation. A new approach for $\lambda_{2}$ was obtained by Zahn 1989 using the mixing-length theory. He obtained

$\lambda_{2}=0.607 \alpha^{4 / 3} E^{2 / 3} \int_{x \mathrm{~b}}^{1} x^{22 / 3}(1-x)^{2} \mathrm{~d} x$

where $x_{\mathrm{b}}$ denotes the bottom of the surface convective zone, $\alpha$ is the mixing-length parameter and $E$ describes a polytropic envelope. I have evaluated it through the equation

$E=\frac{Q_{\text {conv }}}{\int_{x_{\mathrm{b}}}^{1}\left(\frac{2(1-x)}{5 x}\right)^{3 / 2} x^{2} \mathrm{~d} x}$

where $Q_{\text {conv }}$ is the mass of the convective envelope. The approximation described above is valid when the convective turnover time is smaller than the tidal period.

In Fig. 9 we can see the behaviour of the tidal torque constant and tidal coefficient for a $10 M_{\odot}$. Thick continuous line represents the evolution of $E_{2}$, thin continuous line the evolution of $\lambda_{2}$ and dashed line denotes the evolution of $k_{2}$. The tidal torque constant decreases quickly as the model evolves. From homogeneous model to the hydrogen exhaustion it decays about 4 magnitudes. The contribution of the equilibrium tide is very small in the main-sequence, as expected. In this specific case we have computed this contribution only for models with effective temperatures below $8000 \mathrm{~K}$. This contribution increases with the depth of the convective outer layers. A rapid analysis of Fig. 9 during the phases $d-f$ shows that indeed the apsidal motion constant $k_{2}$ is a good approximation to $\lambda_{2}$.

In the next papers of this series we will analyse the influence of the chemical compositions and core overshooting on the $\lambda_{2}$ and $E_{2}$. The present models are available in electronic form at the CDS or directly from the author. Also, I can provide to interested readers specific calculations or details which were not given here.

Acknowledgements. Drs. M.A. López Valverde and G. Meynet are gratefull acknowledged by their useful suggestions. The Spanish DGYCIT (PB93-0134) is gratefully acknowledged for support during the development of this work.

\section{References}

Alexander D.R., 1992 (private communication)

Claret A., 1995, A\&AS 109, 441 (Paper I)

Claret A., Cunha N.C.S., 1997, A\&A 318, 187

Claret A., Giménez A., Cunha N.C.S., 1995, A\&A 299, 724

Cowling T.G., 1941, MNRAS 101, 36

Iglesias C.A., Rogers F.J., Wilson B.G., 1992, ApJ 397, 771

Rieutord M., 1992, A\&A 259, 581

Tassoul J.L., Tassoul M., 1996, Fundament. Cosm. Phys. 16, 377

Zahn J.P., 1975, A\&A 41, 329

Zahn J.P., 1989, A\&A 220, 112 